\title{
Detecting a Sodium Chloride Ion Pair in Ice Using Terahertz Time-domain Spectroscopy
}

\author{
Rakchanok RungSawang, Yuko Ueno, and Katsuhiro AJITO ${ }^{\dagger}$ \\ NTT Basic Research Laboratories, 3-1 Morinosato-Wakamiya, Atsugi, Kanagawa 243-0198, Japan
}

\begin{abstract}
The hydrogen bond resonance of a sodium chloride $(\mathrm{NaCl})$ ion pair trapped in aqueous ice has been observed by transmission terahertz time-domain spectroscopy. The absorption peak of a sodium chloride ion pair in ice is $1.65 \mathrm{THz}$ at $83 \mathrm{~K}$. By investigating the interaction of the cation and anion with other chemical compounds, we deduce that the absorption peak originates from the hydrogen bond resonance of sodium chloride and water molecules. The charge redistribution that occurs when other ion pairs are added to aqueous salt solution changes the absorption spectrum. Furthermore, the results also indicate that simple molecules such as sodium halides have fingerprints in the terahertz region when the ions are trapped in ice. $\mathrm{NaCl}$ ion pairs in seawater and in Ringer's solution were examined.
\end{abstract}

(Received April 15, 2007; Accepted May 31, 2007; Published July 10, 2007)

\section{Introduction}

Sodium chloride $(\mathrm{NaCl})$ is essential for life and appears in most biological tissues and body fluids. For example, the nervous system is regulated by sodium ions. Sodium chloride salt trapped in ice has long been studied because of its great importance to both terrestrial and oceanographic history. ${ }^{1}$ The crystalline structure of ice is typically examined by using incoherent neutron scattering, which provides the density state of vibrations, and is studied theoretically by molecular dynamics simulation. ${ }^{2,3}$ Alternatively, the non-covalent bonds between ions and water molecules could be a clue to the trapping mechanism. However, such weak bonds were difficult to observe because of the need for low frequency radiation sources and a sensitive detection technique. Terahertz timedomain spectroscopy (THz-TDS) has been used for numerous investigations of the low frequency vibrations of various sample forms, including gases, liquids, crystalline powders and ice. This technique provides a far-infrared spectrum with a high signal to noise ratio because of the brightness of the low frequency components of $\mathrm{THz}$ pulses and the coherent detection technique. An absorption peak in the $0.1-4.0 \mathrm{THz}$ range is typically found in organic crystalline samples because of the contribution of intramolecular and non-covalent vibrational modes. ${ }^{4-6}$ Since $\mathrm{THz}$ radiation cannot travel very far in liquid water, reflection spectroscopy is one method for studying its properties in the low frequency region. ${ }^{7}$ With a high power $\mathrm{THz}$ source such as a free electron laser and a sensitive cryogenic detector, the absorption spectrum of water has been observed with an attenuation length of the order of tens of micrometers. ${ }^{8}$ $\mathrm{THz}$ waves propagate further in aqueous ice because there are fewer molecular vibration modes. The smooth spectrum of ice crystals in the $0.25-1.0 \mathrm{THz}$ range measured with THz-TDS

† To whom correspondence should be addressed.

E-mail: ajito@nttbrl.jp

R. R. present address: Semiconductor Physics Group, Cavendish Laboratory, JJ Thomson Ave., Cambridge CB3 0HE, UK. was reported by Zhang et al. ${ }^{9}$ Using inelastic neutron scattering, researchers observed the density of the vibration states of crystalline and amorphous ice, which contribute to the translational, librational and internal vibrational modes, from $0.8-31.4 \mathrm{THz}$ with a frequency resolution of $0.5-2.0 \mathrm{THz} .^{10}$ The density of the vibrational state of amorphous ice has been calculated using molecular dynamics. ${ }^{11}$ Both results are in good agreement: a broad vibrational state density peak appears at 2 $\mathrm{THz}$ caused by transverse acoustic modes. ${ }^{2}$ This prohibits the observation of other resonances overlapping with this frequency region. However, the frequency range below $2 \mathrm{THz}$, where an intense source, i.e., a photoconductive antenna, is available, would be a promising window for observing the weak interactions.

The freezing point of the solution decreases as the salt concentration increases. It is known that salt solubility in liquid water and ice differs because of brine rejection when the salt solution is being frozen. In other words, with a high salt concentration, the ions can be trapped inside the ice crystal. Theoretical studies of brine rejection based on molecular dynamic simulations were reported by Vrbka and Jungwirth. ${ }^{12}$ Sensing the weak interactions in the ice form may provide trapping evidence and one may identify a solution using its farinfrared spectrum.

In this paper, we report the existence of sodium chloride $(\mathrm{NaCl})$ ion pairs trapped in ice observed in a terahertz transmission spectrum. We studied the origin of the strong absorption peaks experimentally. The measurement method was used to investigate $\mathrm{NaCl}$ in a real seawater sample and in Ringer's solution, which has the same concentration as body fluids.

\section{Experimental}

A THz spectrometer (THz-TDS 2004, Aispec, Tokyo) was used to measure the $\mathrm{THz}$ transmission spectra of the samples. The emitter and detector of the $\mathrm{THz}$ pulses were micro-gap photoconductive antennas. Optical pulses (100 fs) from a 


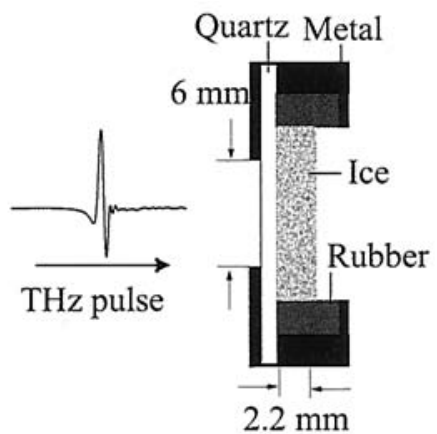

Fig. 1 Side view of ice sample in holder.

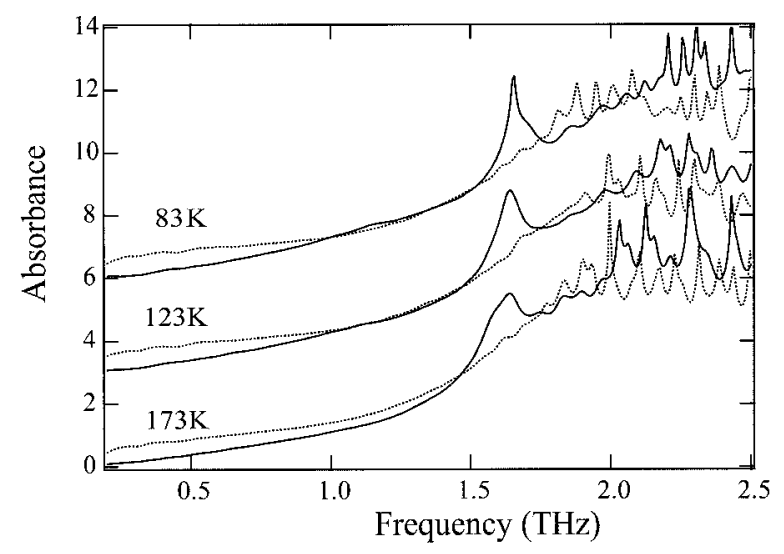

Fig. 2 Absorption spectra of distilled water ice (dotted lines) and aqueous $\mathrm{NaCl}$ ice (solid lines) at different temperatures. The $\mathrm{NaCl}$ solution concentration was $0.77 \mathrm{~mol} / \mathrm{L}$. The spectra for 123 and $83 \mathrm{~K}$ are vertically offset by a value of two.

Ti-sapphire laser (Vitesse, Coherent, California) were transmitted to pulse compression components. After being compressed to 40 -fs pulse duration, the optical beam was sent to the photoconductive antennas for $\mathrm{THz}$ generation and detection. The $\mathrm{THz}$ beam was focused on a sample mounted in a liquid nitrogen flow cryostat. The sample holder in the cryostat was composed of a metal aperture $6 \mathrm{~mm}$ in diameter, a 1-mm thick quartz plate, a rubber ring with an 8-mm inner diameter and a metal cover. A schematic diagram of the sample holder is shown in Fig. 1. We used only one quartz plate in order to minimize absorption loss. THz pulses were transmitted through the metal aperture, the quartz plate and the ice sample, respectively.

\section{Sample Preparation}

$\mathrm{NaCl}$ and other powder samples were obtained from SigmaAldrich (Tokyo). The sample powder was dissolved in distilled water. A $90-\mu \mathrm{L}$ volume of the solution was then poured into the sample holder. More specifically, the sample holder without ice seen in Fig. 1 was rotated $90^{\circ}$ counterclockwise to fill it with liquid sample. Then the sample holder was inserted into the chamber with a nitrogen gas flow to prevent water vapor absorption inside the chamber. The sample cooling rate was controlled by mechanically pumping liquid nitrogen at a flow rate of $6.0 \mathrm{dm}^{3} / \mathrm{min}$ so that the ice was formed very quickly. As a result, the non-uniformity of the ions in the ice can be

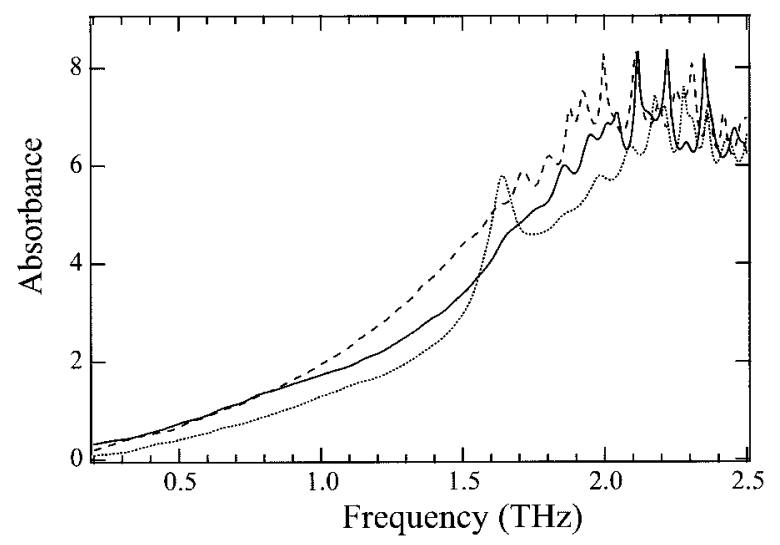

Fig. 3 Comparison of absorption spectra of alkali chlorides at 123 $\mathrm{K}$; $\mathrm{LiCl}$ (dashed line), $\mathrm{NaCl}$ (dotted line), and $\mathrm{KCl}$ (solid line) with a concentration of $0.77 \mathrm{~mol} / \mathrm{L}$.

suppressed. If the solution is frozen slowly, sodium chloride salt will be rejected into the unfrozen part of the system. One surface of the ice sample was not ideally flat. The ice thickness was approximately $2.2 \mathrm{~mm}$ at $123 \mathrm{~K}$. After the sample became ice, the sample holder was rotated $90^{\circ}$ so that $\mathrm{THz}$ pulses could pass through the quartz plate and ice, respectively, as shown in Fig. 1. Then the chamber was pumped out to reduce water vapor absorption during the measurement.

Seawater was taken from about one meter below the sea surface at a point off the Izu peninsula in the south east of Japan. The water was then filtered with $1 \mu \mathrm{m}$ grids. We used Ringer's solution purchased from Fuso Ltd. (Tokyo); this contained $0.86 \% \mathrm{NaCl}, 0.3 \% \mathrm{KCl}$, and $0.033 \% \mathrm{CaCl}_{2}$, namely the $\mathrm{NaCl}$ concentration was $0.147 \mathrm{~mol} / \mathrm{L}$.

\section{Results}

Figure 2 shows the absorption spectra of aqueous ice and aqueous $\mathrm{NaCl}$ ice at different temperatures. Aqueous ice has a smooth spectrum up to $1.8 \mathrm{THz}$, where it begins to roughen because of the darkness of the high frequency components and the high-density vibration modes of ice. On the other hand, aqueous $\mathrm{NaCl}$ ice has a strong absorption around $1.6 \mathrm{THz}$ at 123 $\mathrm{K}$. The peak becomes narrower and shifts to a higher frequency with decreasing temperature as the lattice shrinks and the thermal broadening decreases. The peak positions are 1.642, 1.642 , and $1.656 \mathrm{THz}$ from the high temperatures to the low temperatures, respectively. The spectrum of a $\mathrm{NaCl}$ solution with double the concentration (not shown here) provided strong absorption starting from $1.64 \mathrm{THz}$ and continuing, so we could not distinguish an absorption peak at this high concentration.

We studied the anion $\mathrm{Cl}^{-}$to investigate the origin of the absorption peak in $\mathrm{NaCl}$ ice. Alkaline chlorides were dissolved in water at the same concentration and tested using the method described above. The absorption spectra of $\mathrm{LiCl}$ and $\mathrm{KCl}$ shown in Fig. 3 were compared with that of $\mathrm{NaCl}$. The results indicate that there is no particular absorption caused by lithium or potassium chloride in the observed range.

We studied the effect of other halogenic anions, i.e., $\mathrm{F}, \mathrm{Br}$ and $\mathrm{I}$, on the spectrum of sodium compounds. $\mathrm{NaF}$ and $\mathrm{NaI}$ were solved in water with a concentration of $1.54 \mathrm{~mol} / \mathrm{L}$, while the $\mathrm{NaBr}$ solution had half the $\mathrm{NaCl}$ concentration to prevent strong absorption. The spectra in Fig. 4 show that sodium ions might be the origin of the $\mathrm{NaCl}$ absorption peak. However, the 


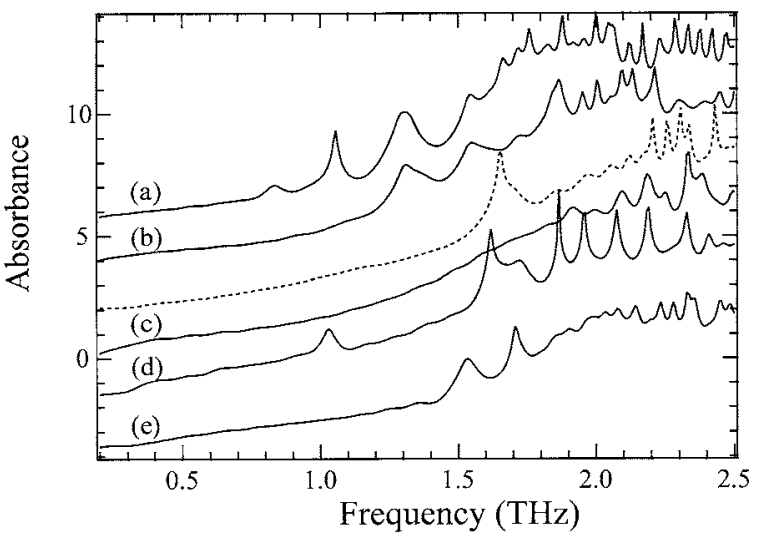

Fig. 4 Absorption spectra of (a) $\mathrm{NaI} 1.54 \mathrm{~mol} / \mathrm{L}$, (B) $\mathrm{NaBr} 0.77$ $\mathrm{mol} / \mathrm{L}$, (c) $\mathrm{NaF} 1.54 \mathrm{~mol} / \mathrm{L}$, (d) $\mathrm{MgCl}_{2} 1.02 \mathrm{~mol} / \mathrm{L}$, (e) $\mathrm{Na}_{2} \mathrm{SO}_{4} 1.54$ $\mathrm{mol} / \mathrm{L}$, and $\mathrm{NaCl}$ (dashed line), shown in Fig. 2, at $83 \mathrm{~K}$. The spectra are vertically offset from each other by a value of two. Peaks above $1.8 \mathrm{THz}$ are artifacts.

Table 1 Peak positions (THz) of samples in Fig. 4

\begin{tabular}{ccccc}
\hline $\mathrm{NaI}$ & $\mathrm{NaBr}$ & $\mathrm{NaCl}$ & $\mathrm{MgCl}_{2}$ & $\mathrm{Na}_{2} \mathrm{SO}_{4}$ \\
\hline 0.832 & & & & \\
1.056 & & & 1.027 & \\
1.309 & 1.309 & & & \\
1.548 & 1.548 & & & 1.534 \\
& & 1.656 & 1.620 & \\
& 1.729 & & 1.722 & 1.707 \\
\hline
\end{tabular}

number of peaks increased with the increasing molecular weight of the halide while $\mathrm{NaF}$ exhibits a smooth spectrum. The peak positions of the spectra in Fig. 4 are shown in Table 1.

A concentration-dependent feature of $\mathrm{NaCl}$ was observed. The concentration was varied so as to take values of 0.225 , $0.449,0.674$, and $0.992 \mathrm{~mol} / \mathrm{L}$. The absorption intensity increases with increasing concentration as shown in Fig. 5(a). The inset depicts the plot of absorbance versus concentration. In the same figure, the seawater spectrum is shown as (b). We can see that the spectrum is not as smooth as the aqueous spectrum around $1.65 \mathrm{THz}$. The Ringer's solution, spectrum plotted in Fig. 5(c) has a detectable peak at $1.67 \mathrm{THz}$.

\section{Discussion}

Since the aqueous solution cannot be observed by THz-TDS because of the resonance vibrations of water, cooling the solution is a promising method and can also provide fingerprints of simple chemical compounds such as $\mathrm{NaCl}$. However, different cooling speeds can lead to different peak positions or to the disappearance of the peak because of the formation of a crystal ice structure. The ice structure forms freely in the $\mathrm{THz}$ pulse propagation direction. Since the sample surface was flat only on the side with the quartz plate, the roughness of the back surface may cause changes in absorption. In our experiment, we found that the roughness affected the peak position of the order of a few times the spectral resolution, namely $7.233 \mathrm{GHz}$.

The $\mathrm{NaCl}$ absorption peak in Fig. 2 became sharper and shifted to higher frequencies because of the reduction in the vibration modes as a result of heat and the shrinking of the bonds. If the absorption peak originates solely from $\mathrm{O}-\mathrm{H}^{\circ} \cdot \mathrm{Cl}^{-}$,

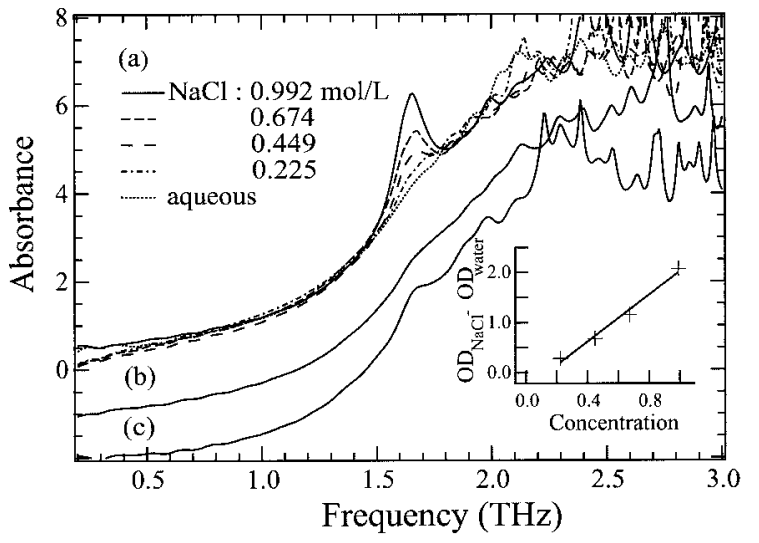

Fig. 5 Absorbance of (a) $\mathrm{NaCl}$ at different concentrations and water (dotted line), (b) seawater, and (c) Ringer's solution at $123 \mathrm{~K}$. Inset is the optical density (OD) of $\mathrm{NaCl}$ above that of water at 1.656 $\mathrm{THz}$ versus concentration (crossed signs) along with a fitting curve $y=-0.3206+2.3446 x$. Here, $y$ is the difference between $\mathrm{NaCl}$ absorbance $\left(\mathrm{OD}_{\mathrm{NaCl}}\right)$ and water $\left(\mathrm{OD}_{\text {water }}\right)$ absorbance, and $x$ is concentration.

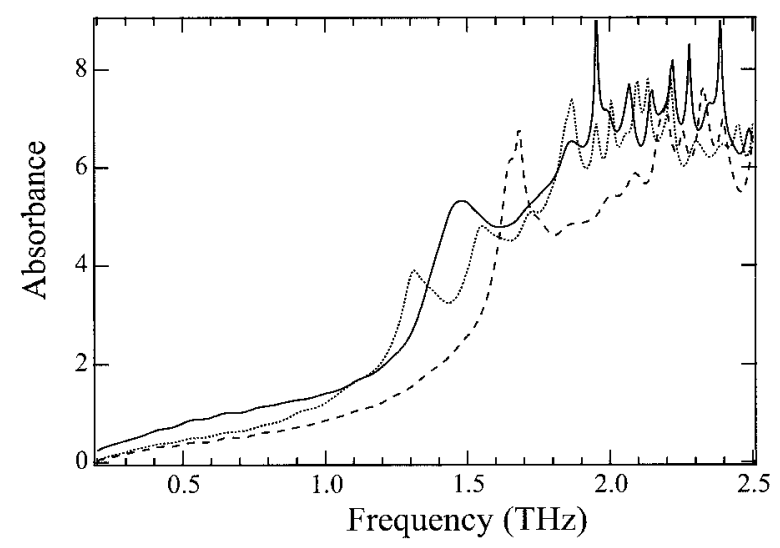

Fig. 6 Absorption spectra of mixed solution, solid line, of 0.77 $\mathrm{mol} / \mathrm{L} \mathrm{NaCl}$ (dashed line) and $0.77 \mathrm{~mol} / \mathrm{L} \mathrm{NaBr}$ (dotted line) with a ratio of $1: 1$. The spectra were obtained at $83 \mathrm{~K}$.

the peak should appear in $\mathrm{KCl}$ and $\mathrm{LiCl}$ ice. But the experimental results in Fig. 3 indicate that only hydrogen bonds with $\mathrm{NaCl}$ are detectable. By observing other sodium halides, we found other absorption peaks. Interestingly, the number of absorption peaks increases with the size of the halogenic ions. This means that the larger halides reveal a hydrogen-bonding network of water molecules, whereas the spectrum of $\mathrm{NaF}$ ion pairs in ice did not show such hydrogen-bonding features. However, we found no correlation of anions. We performed another experiment to observe the interaction of halogenic ions with mixed solutions. $\mathrm{NaCl}$ and $\mathrm{NaBr}$ solutions of $0.77 \mathrm{~mol} / \mathrm{L}$ were mixed together with the same ratio. The absorption peak of this sample, displayed as a solid line in Fig. 6, is broad with a center at $1.468 \mathrm{THz}$, and does not show the convolution of the individual solutions. We tested another mixed solution, i.e. $\mathrm{NaCl}$ and maleic acid $\left(\mathrm{C}_{4} \mathrm{H}_{4} \mathrm{O}_{4}\right)$, but the result is not shown here. The peaks of the individual solutions appeared in the mixed spectrum without any significant change in peak position. From such results, we can consider that there is an interaction between $\mathrm{NaCl}$ and $\mathrm{NaBr}$ ion pairs that causes a charge redistribution. As a result, the hydrogen bonding was modified. The absorbance of $\mathrm{NaCl}$ is linearly proportional to the 
concentration as shown in the inset of Fig. 5.

The fact that the water molecules exhibit a different charge distribution to an ion than to another water molecule is physically reasonable. The absorption of $\mathrm{NaCl}$ can be observed in natural seawater. This implies that the THz-TDS of ice might be applicable to oceanographic research. Furthermore, it is a non-destructive method for detecting $\mathrm{NaCl}$ at the level that appears in human fluids. However, a high concentration of other ions may affect the $\mathrm{NaCl}$ peak position. The amount of $\mathrm{NaCl}$ in meat can be determined by near-infrared diffuse reflectance spectroscopy. At the same time, THz-TDS could be useful for measuring the amount of salt in frozen samples.

\section{Conclusion}

We report the weak interaction of ions trapped in aqueous ice in the $0.2-2.0 \mathrm{THz}$ range observed with THz-TDS. The absorption peaks reflect hydrogen bond vibrations in the particular crystalline structure in the presence of ions because no correlation was found between cations and anions. A theoretical calculation is necessary to explain the origin of resonant peaks. However, this study is a preliminary experimental study showing the far-infrared response that might reflect the structure of ions incorporated in ice. Furthermore, we showed that $\mathrm{THz}$ spectroscopy is sensitive to the quantity of $\mathrm{NaCl}$ ion pairs present in actual bimolecular samples such as Ringer's solution. The observation of weak interactions of ions and water molecules with THz-TDS might open the way to experimental studies on molecule orientations in ice. Furthermore, the method could be adopted for food analysis.

\section{Acknowledgements}

We acknowledge the Wakate Project at NTT for financial support and Dr. Keiichi Torimitsu of NTT for his encouragement.

\section{References}

1. L. Couture and A. Le Paillier-Maléco, J. Phys. C: Solid State Phys., 1975, 8, L67.

2. H. J. Prask, S. F. Trevino, J. D. Gault, and K. W. Logan, J. Chem. Phys., 1972, 56, 3217

3. M. Matsumoto, S. Saito, and I. Ohmine, Nature, 2002, 416, 409.

4. B. Fisher, M. Hoffmann, H. Helm, G. Modjesch, and P. U. Jepsen, Semicond. Sci. Technol., 2005, 20, 246.

5. Y. Ueno, R. Rungsawang, I. Tomita, and K. Ajito, Chem. Lett., 2006, 35, 1128.

6. B. Yu, F. Zeng, Y. Yang, Q. Xing, A. Chechin, X. Xin, I. Zeylikovich, and R. R. Alfano, Biophys. J., 2004, 86, 1649.

7. C. Rønne, L. Thrane, P. Åstrand, A. Wallqvist, K. V. Mikkelsen, and S. R. Keiding, J. Chem. Phys., 1997, 107, 5319.

8. J. Xu, K. W. Plaxco, and S. J. Allen, J. Chem. Phys., 2006, $124,036101$.

9. C. Zhang, K. Lee, X.-C Zhang, X. Wei, and Y. R. Shen, Appl. Phys. Lett., 2001, 79, 491.

10. D. D. Klug, E. Whalley, E. C. Svensson, J. H. Root, and V. F. Sears, Phys. Rev. B: Condens. Matter Mater. Phys., 1991, 44, 841 .

11. J. S. Tse, D. D. Klug, C. A. Tulk, E. C. Svensson, I. Swainson, V. P. Shpakov, and V. R. Belosludov, Phys. Rev. Lett., 2002, 85, 3185.

12. L. Vrbka and P. Jungwirth, Phys. Rev. Lett., 2005, 95, 148501. 